\title{
TOPOLOGICAL COMPLETIONS OF METRIZABLE SPACES
}

\author{
BEN FITZPATRICK, JR., GARY F. GRUENHAGE, AND JAMES W. OTT
}

(Communicated by Dennis Burke)

\begin{abstract}
For a pair of metrizable spaces $X$ and $Y$, we investigate conditions under which there is a dense embedding $h: X \rightarrow Z$, where $Z$ is completely metrizable and $Z \backslash h(X)$ is homeomorphic to $Y$. In such a case, $Z$ is called a topological completion of $X$ and $Y$ is called a completion remainder of $X$. In case $X$ and $Y$ are completely metrizable, we give necessary and sufficient conditions that $Y$ be a completion remainder of $X$. We characterize the completion remainders of $\mathbf{R}$ and those of the rationals, $\mathbf{Q}$. We also characterize the remainders of $\mathbf{Q}(\kappa)$, a nonseparable analogue of $\mathbf{Q}$.
\end{abstract}

\section{INTRODUCTION}

Wilanski [4] asked whether there is a 3-point completion of the reals, i.e., is there a dense embedding $h$ of $\mathbf{R}$ into a Polish space $Z$ such that $|Z \backslash h(\mathbf{R})|=$ 3 ? More generally, one might ask under what conditions on metrizable spaces $X$ and $Y$ does there exist a homeomorphism $h$ of $X$ into a completely metrizable space $Z$ such that $h(X)$ is dense in $Z$ and $Z \backslash h(X)$ is homeomorphic to $Y$. In such a case, $Z$ is called a topological completion of $X$ and $Y$ is called a completion remainder of $X$. In case $h(X)$ is open in $Z, Y$ is called a closed completion remainder of $X$. Throughout, if $X$ is a space, $d(X), w(X)$, and $e(X)$ denote, respectively, the density, weight, and extent of $X$ and $l d_{p}(x)$ denotes the local density at $p$ of $X$ :

$$
\begin{aligned}
& d(X)=\omega+\inf \{|M|: M \text { is dense in } X\} \\
& w(X)=\omega+\inf \{|\mathscr{B}|: \mathscr{B} \text { is a basis for } X\} ; \\
& e(X)=\omega+\sup \{|D|: D \text { is a closed, discrete set in } X\} ; \\
& l d_{p}(X)=\omega+\inf \{|D|: D \text { is dense in some open } U \text { in } X, p \in U\} .
\end{aligned}
$$

For metrizable spaces, $d(X)=w(X)=e(X)$. This and other relevant properties are to be found in Engelking [1].

In $\S 2$ we characterize those pairs $(X, Y)$ of completely metrizable spaces such that $Y$ is a closed completion remainder of $X$ and those pairs such that $Y$ is a completion remainder of $X$. Section 3 provides a characterization of the completion remainders of $\mathbf{Q}$, the rationals, and gives both necessary and sufficient conditions (neither being necessary and sufficient) for a space to

Received by the editors August 7, 1990 and, in revised form, May 6, 1991.

1991 Mathematics Subject Classification. Primary 54E99.

Key words and phrases. Completely metrizable, topological completions, Polish spaces, $\sigma$-compact, $\sigma$-discrete, nowhere locally compact. 
be a completion remainder of $\mathbf{P}$, the irrationals. Section 4 studies completion remainders of nonseparable analogs $\mathbf{Q}(\kappa)$ and $\mathbf{P}(\kappa)$ of $\mathbf{Q}$ and $\mathbf{P}$. We conclude in $\S 5$ with two open questions.

\section{THE COMPLETELY METRIZABLE CASE}

It is easy to see that if $X$ and $Y$ are metrizable and $Y$ is a completion remainder of $X$, then $d(Y) \leq d(X)$ and that if $Y$ is nonempty then $X$ is not compact. Whether or not $e(X)$ is achieved, that is, whether there exists a closed discrete set in $X$ whose cardinality is $e(X)$, plays an important role. Note that, for metrizable spaces $X$, if $e(X)$ is not achieved then $e(X)$ has countable cofinality. We begin with an example.

Example 1. A completely metrizable space $Z=X \cup Y$, where $X \cap Y=\varnothing, X$ is dense in $Z$, and there is no closed discrete set in $X$ of cardinality $d(Y)$. Consider a hedgehog $H$, centered at a point $\mathscr{O}$, where $H=\bigcup_{i<\omega} H_{i}$ and each $H_{i}$ has $\aleph_{i}$ spines of length 1 . That is, $H_{i}=\{\mathscr{O}\} \cup \bigcup_{\lambda \in \Lambda_{i}}((0,1] \times\{\lambda\})$, where $\Lambda_{i}$ is an indexing set of cardinality $\aleph_{i}$. Take $\Lambda_{i} \cap \Lambda_{j}=\varnothing$ for $i \neq j$. Let $H_{i}^{\prime}=\{\mathscr{O}\} \cup \bigcup_{\lambda \in \Lambda_{i}}\left(\left(0,2^{-i}\right] \times\{\lambda\}\right)$; let $Z=\bigcup_{i<\omega} H_{i}^{\prime}$; let $Y=\left\{\left\langle 2^{-i}, \lambda\right\rangle: i<\omega\right.$, $\left.\lambda \in \Lambda_{i}\right\}$; and let $X=Z \backslash Y$. Note that $Y$ is a discrete space of cardinality $\aleph_{\omega}$ but every discrete subset of $X$ of cardinality $\aleph_{\omega}$ has $\mathscr{O}$ as a limit point.

This example motivates the following useful lemma.

Lemma 1. Suppose $X$ is a metrizable space and $e(X)$ is not achieved. Then there exists a point $x$ of $X$ such that $l d_{x}(X)=e(X)$. Moreover, the set of all such points is compact.

Proof. Assume that $e(X)$ is not achieved. Let $\alpha_{-1}=0$. Let $\left\{\alpha_{n}: n<\omega\right]$ be an increasing sequence of cardinals whose sum is $e(X)$. Suppose $l d_{p}(X)<e(X)$ for all $p \in X$. There is a minimal, locally finite open cover $\mathscr{U}$ of $X$ such that for all $U \in \mathscr{U}, d(U)<e(X)$. Then $|\mathscr{U}|<e(X)$. If there is a cardinal $\alpha<e(X)$ such that $d(U)<\alpha$ for all $U \in \mathscr{U}$, then $d(X) \leq \alpha \cdot|\mathscr{U}|<e(X)$, which is impossible. For each $n<\omega$ there is a $U_{n} \in \mathscr{U}$ such that $d\left(U_{n}\right) \geq \alpha_{n}$. Since $d\left(U_{n}\right)=d\left(\bar{U}_{n}\right)=e\left(\bar{U}_{n}\right)$, there is a closed discrete set $D_{n}$ in $\bar{U}_{n}$ such that $\left|D_{n}\right| \geq \alpha_{n-1}$. Then $D=\bigcup_{n \in \omega} D_{n}$ is closed and discrete and has cardinality $e(X)$, a contradiction.

Next, assume that there is an infinite closed and discrete set $A=\left\{a_{n}: n<\omega\right\}$ such that $l d_{a}(X)=e(X)$ for every $a \in A$. There is a discrete collection $\left\{U_{n}: n<\omega\right\}$ of open sets screening $A$. For each $n<\omega$, there is a closed discrete set $D_{n}$ in $\bar{U}_{n}$ of cardinality $\geq \alpha_{n-1}$. Then $D=\bigcup_{n<\omega} D_{n}$ is closed and discrete in $X$ and has cardinality $e(X)$, which is impossible.

Theorem 1. Let $X$ and $Y$ be completely metrizable spaces. Then $Y$ is a closed completion remainder of $X$ if and only if there is a closed discrete subset of $X$ of cardinality $d(Y)$.

Proof. Suppose first that $Y$ is a closed completion remainder of $X, h: X \rightarrow Z$ is a dense embedding, $Z$ is completely metrizable, $Z \backslash h(X)$ is closed in $Z$ and is homeomorphic to $Y$. Since $d(Y) \leq d(X)=e(X)$, it follows that if $e(X)$ is achieved or if $d(Y)<e(X)$ then there is a closed discrete set in $X$ of cardinality $d(Y)$. Assume then that $d(Y)=e(X)$ and that $e(X)$ is not achieved. The set $K$ of all points of $X$ at which $X$ has local density $e(X)$ is 
compact. Thus $h(K)$ and $Z \backslash h(X)$ are disjoint closed sets in $Z$ and can be enclosed in open sets $U_{X}$ and $U_{Y}$ with disjoint closures. Let

$$
Z^{\prime}=Z \cap \bar{U}_{Y}, \quad X^{\prime}=h(X) \cap \bar{U}_{Y}, \quad Y^{\prime}=(Z \backslash h(X)) \cap \bar{U}_{Y} .
$$

Then $Z^{\prime}$ is completely metrizable, $Y^{\prime} \simeq Y, Y^{\prime}$ is closed in $Z^{\prime}$, and $X^{\prime}$ is dense in $Z^{\prime}$. Now, since $X^{\prime} \cap U_{X}=\varnothing$, then for all $p \in X^{\prime}$ we have $l d_{p}\left(X^{\prime}\right)<e(X)=e(Y)+e\left(X^{\prime}\right)$; therefore $e\left(X^{\prime}\right)$ is achieved. Let $D$ be a closed discrete set in $X^{\prime}$ of cardinality $e(X)$. Then $h^{-1}(D)$ is closed and discrete in $X$.

Next, assume that $X$ has a closed discrete subset of cardinality $d(Y)=\alpha$. For some (perhaps finite) cardinal $\beta, Y$ has a dense subset $K$ of cardinality $\beta$. We further assume that $X \cap Y=\varnothing$ (otherwise, take disjoint copies $X^{\prime}$ and $\left.Y^{\prime}\right)$. Since $\alpha$ is infinite and $\beta \leq \alpha$, there is a discrete collection $\mathscr{H}$ of open sets in $X$ of cardinality $\beta \cdot \omega$. Let $H$ be an Axiom of Choice set for $\mathscr{H}$, and let $T$ be the induced mapping from $H$ onto $\mathscr{H}$. Now, $H=\bigcup_{n<\omega} H_{n}$, where $H_{m} \cap H_{n}=\varnothing$ for $m \neq n$, and $\left|H_{n}\right|=\beta, n<\omega$. For each $n$, let $T_{n}$ denote a bijection from $K$ onto $H_{n}$.

There exists a sequence $\left\{G_{n}^{\prime}: n<\omega\right\}$ for $X$ as in Moore's metrization theorem [3], i.e., for each $n, G_{n}^{\prime}$ is an open covering of $X, G_{n+1}^{\prime} \subseteq G_{n}^{\prime}$, and for every $p \in X,\left\{S t^{2}\left(G_{n}^{\prime}, p\right): n<\omega\right\}$ forms a local base for the topology at $p$. For each $p \in H$, denote by $R_{0}(p)$ an element of $G_{0}^{\prime}$ containing $p$ whose closure is a subset of $T(p)$ and, having defined $R_{n-1}(p)$, denote by $R_{n}(p)$ an element of $G_{n}^{\prime}$ containing $p$ whose closure is a subset of $R_{n-1}(p)$. For each $n<\omega$, let $G_{n}$ be the collection of all elements $g$ of $G_{n}^{\prime}$ such that if $p \in H$ and $i \leq n$ then $g$ does not intersect both $R_{i}(p)$ and $X \backslash R_{i-1}(p)$.

Let $\rho$ denote a metric on $Y$. For $y \in Y$ and $\delta>0$ let $B(y, \delta)=\{z \in Y$ : $\rho(z, y)<\delta\}$. If $q \in Y$ and $k<\omega$, define

$$
E_{k}(q)=B\left(q, 2^{-k}\right) \cup \bigcup\left\{R_{k}\left(T_{j}(s)\right): k \leq j<\omega \text { and } s \in B\left(q, 2^{-k}\right) \cap K\right\} .
$$

For $i<\omega$ define $M_{i}=\left\{E_{j}(q): q \in Y\right.$ and $\left.j \geq i\right\}$. Let $L_{i}=M_{i} \cup G_{i}$, and let $Z=X \cup Y$. Then $L_{0}$ is a basis for a $T_{1}$-topology $\Omega$ on $Z$ and $\left\{L_{n}: n<\omega\right\}$ satisfies the conditions of Moore's theorem, so that $(Z, \Omega)$ is metrizable. Clearly, the inclusion maps $\Phi_{x}: X \rightarrow Z$ and $\Phi_{Y}: Y \rightarrow Z$ are homeomorphisms, $X$ is a dense open set in $Z$, and $Z \backslash X=Y$.

Now, let $Z^{\prime}$ be a completely metrizable space containing $Z$. Since $X$ and $Z \backslash X$ are completely metrizable, they are $G_{\delta}$-sets in $Z^{\prime}$. The union of two $G_{\delta}$-sets is a $G_{\delta}$-set, so $Z$ is a $G_{\delta}$-set in the complete space $Z^{\prime}$ and is itself complete.

Corollary 1. If $X$ and $Y$ are completely metrizable, $X$ is not compact, and $Y$ is separable, then $Y$ is a completion remainder of $X$.

Remark. It is clear that if $X$ and $Y$ are metrizable and there is a dense embedding $h$ of $X$ into a completely metrizable space $Z$ such that $Z \backslash h(X) \simeq Y$ and such that $h(X)$ is open in $Z$, then $X$ and $Y$ must be completely metrizable. It is also clear that the metrizable space $X$ is an absolute $F_{\sigma}$ if and only if every completion remainder of $X$ is complete. Thus we have

Corollary 2. The completion remainders of $\mathbf{R}$, or, indeed, of any separable, locally compact, noncompact, metrizable space, are the nonempty Polish spaces. 
Corollary 3. Suppose $X$ and $Y$ are completely metrizable and $X$ is not compact. Let $e(X)$ be achieved, i.e., let $X$ have a closed, discrete set of cardinality $e(X)$. Then the following are equivalent.

(A) $d(Y) \leq e(X)$.

(B) $Y$ is a completion remainder of $X$.

(C) $Y$ is a closed completion remainder of $X$.

Theorem 2. Suppose $X$ and $Y$ are completely metrizable and $X$ is not compact. Suppose $e(X)$ is not achieved. Then $Y$ is a completion remainder of $X$ if and only if $d(Y) \leq e(X)$ and $l d_{y}(Y)<e(X)$ for every $y \in Y$.

Proof. Let $\left\{\alpha_{n}: n<\omega\right\}$ be an increasing sequence of cardinals whose supremum is $e(X)$. As before, we can assume that $X \cap Y=\varnothing$. Suppose first that $Z$ is completely metrizable, $Z=X \cup Y$, and $X$ is dense in $Z$. Suppose there is a point $y \in Y$ such that $l d_{y}(Y)=e(X)$. For each open set $U$ in $Z$ containing $y, d(U \cap X)=e(X)$. Take a sequence $\left\{U_{n}: n<\omega\right\}$ of open sets in $Z$, $\overline{U_{n+1}} \subset U_{n}, y \in U_{n}$ for every $n<\omega$, and $\operatorname{diam}\left(U_{n}\right)<2^{-n}$. For each $n<\omega$, there is a closed discrete set $D_{n}$ in $X \cap \overline{U_{n}},\left|D_{n}\right|=\alpha_{n}$. Then $D=\bigcup_{n<\omega} D_{n}$ is closed and discrete in $X$ and $|D|=e(X)$, a contradiction. This completes the necessity proof.

Next, suppose $d(Y) \leq d(X)$ and $l d_{y}(Y)<e(X)$ for all $y \in Y$. As before, we assume that $X \cap Y=\varnothing$. We first consider the special case in which $Y=$ $\bigcup_{n<\omega} Y_{n}$ is a countable discrete union of closed subsets, each of density less than $e(X)$. We show in this case that $Y$ is a completion remainder of $X$ in such a way that in the completion $Z=X \cup Y$, the sequence $\left\{Y_{n}: n<\omega\right\}$ converges to a point $p$ of $X$.

Let $p$ be a point of $X$ such that $l d_{p}(X)=d(X)$. Let $U_{0}$ be an open set in $X$ containing $p$ with $\operatorname{diam} U<2^{-0}$. There is a closed discrete set $D_{0}$ in $X, D_{0} \subseteq U_{0}, p \notin D_{0},\left|D_{0}\right|=\alpha_{0}$. There exists a discrete collection $\mathscr{G}_{0}$ of open sets screening $D_{0} \cup\{p\}$ such that $\bigcup\left\{\bar{G}: G \in \mathscr{G}_{0}\right\} \subset U_{0}$. Let $G_{0, p}$ be the element of $\mathscr{G}_{0}$ containing $p$. Having chosen $U_{n-1}, D_{n-1}, \mathscr{G}_{n-1}$, and $G_{n-1, p}$, take $U_{n}$ to be an open set in $X, p \in U_{n}, \operatorname{diam} U_{n}<2^{-n}, \bar{U}_{n} \subset G_{n-1, p}$; take $D_{n}$ to be a closed discrete set in $X, D_{n} \subseteq U_{n}, p \notin D_{n},\left|D_{n}\right|=\alpha_{n}$; take $\mathscr{G}_{n}$ to be a discrete collection of open sets screening $D_{n} \cup\{p\}$ such that $\bigcup\left\{\bar{G}: G \in \mathscr{G}_{n}\right\} \subseteq U_{n}$; and let $G_{n, p}$ denote the element of $\mathscr{G}_{n}$ containing $p$.

As in the sufficiency proof of Theorem 1, there is a topology $T_{n}$ on $\left(U_{n} \mid \bar{U}_{n+1}\right)$ $\cup Y_{n}$ such that $Y_{n}$ is closed and nowhere dense in $\left(U_{n} \backslash \bar{U}_{n+1}\right) \cup Y_{n}$, and where $\mathscr{G}_{n}$ plays the role of the discrete collection $\mathscr{H}$. This latter condition implies that there is a base for $T_{n}$ that is $\sigma$-discrete in $X$. For each $n<\omega$, let $U_{n}^{*}=U_{n} \cup \bigcup\left\{Y_{n}: m \geq n\right\}$. Then $T=\left(\bigcup_{n<\omega} T_{n}\right) \cup\left\{U_{n}^{*}: n<\omega\right\} \cup\{U: U$ open in $X, p \notin U\}$ is a basis for a completely metrizable topology on $X \cup Y$, $X \backslash\{p\}$ is dense and open in $X \cup Y$, and $\left\{Y_{n}: n<\omega\right\}$ converges in $X \cup Y$ to $\{p\}$.

We now proceed to the general case. There is a minimal, locally finite open cover $\mathscr{U}$ of $Y$ such that if $U \in \mathscr{U}$ then $d(U)<e(X)$. Now, $|\mathscr{U}| \leq d(Y) \leq$ $e(X)$, so $\mathscr{U}$ is a countable union, $\mathscr{U}=\bigcup_{n<\omega} \mathscr{U}_{n}$, where $\left|\mathscr{U}_{n}\right| \leq \alpha_{n}$. Let $\mathscr{U}_{n, m}=\left\{U \in \mathscr{U}_{n}: d(U) \leq \alpha_{m}\right\}$, and let $U_{n, m}=\bigcup \mathscr{U}_{n, m}$. Note that $d\left(U_{n, m}\right) \leq$ $\alpha_{n} \cdot \alpha_{m}<e(X)$. By Engelking [1, Lemma 5.2.4], the countable open cover 
this cover can be shrunk, $\left\{\mathrm{Cl}\left(V_{n}\right): n<\omega\right\}$ can be taken to be a star-finite cover of $Y$. For $n<\omega$, let $Y_{n}=\bar{V}_{n}$, let $\left\{Y_{n}^{\prime}: n<\omega\right\}$ be a sequence of disjoint spaces, $Y_{n}^{\prime} \simeq Y_{n}$, and let $Y^{*}$ be the free union of the $Y_{n}^{\prime}$ 's. Then there is a completely metrizable topology on $X \cup Y^{*}$ as in the special case, with $\left\{Y_{n}^{\prime}: n<\omega\right\}$ converging to a point $p \in X$. Let $f: X \cup Y^{*} \rightarrow X \cup Y$ be the obvious quotient map. Note that $f^{-1}(q)$ is finite for all $q \in X \cup Y$.

We claim that $f$ is a closed and therefore perfect mapping. For, let $H \subseteq$ $X \cup Y^{*}$ be closed. If $p \notin H$ then $H \cap Y_{n}^{\prime}=\varnothing$ for all sufficiently large $n$. Since $f^{-1}(f(H))=H \cup \bigcup_{n, m<\omega} f^{-1}\left(f\left(H \cap Y_{n}^{\prime}\right) \cap Y_{m}\right)$, it follows that $f(H)$ is closed in this case. But it is also clearly closed if $p \in H$. Thus $X \cup Y$ is completely metrizable, since it is a perfect image of a completely metrizable space. Clearly, $X$ is dense in $X \cup Y$.

\section{Completion Remainders of $\mathbf{Q}$ AND of $\mathbf{P}$}

Theorem 3. The completion remainders of $\mathbf{Q}$ are the nowhere locally compact Polish spaces.

Proof. Assume $Y$ is a nowhere locally compact Polish space, regarded as a subset of the Hilbert cube. Let $K=\bar{Y}$. Then $Y$ is a dense $G_{\delta}$-set in the compact metric space $K$, and, since $Y$ is nowhere locally compact, $K \backslash Y$ is dense in $K$. Let $G=\left\{G_{n}: n<\omega\right\}$ be a countable basis for $K$. For $n<\omega$, let $U_{n}$ be open in $K$, with $Y=\bigcap_{n<\omega} U_{n}, U_{n} \supseteq U_{n+1}$. Choose $a_{n} \in\left(U_{n} \backslash Y\right) \cap G_{n}$. Let $A=\left\{a_{n}: n<\omega\right\}$. Then $A$, being a countable metric space with no isolated points, is homeomorphic to $\mathbf{Q}$. Let $Z=A \cup Y$. Then $A$ is dense in $Z$. It remains to be shown that $Z$ is completely metrizable. We show that $Z$ is a $G_{\delta}$-set in $K$. Let $V_{n}=U_{n} \cup\left\{a_{i}: i<n\right\}$. Each $V_{n}$, as the union of two $G_{\delta}$-sets, is a $G_{\delta}$-set, so $V_{n}$ is one and $Z=\bigcap_{n<\omega} V_{n}$ is thus a $G_{\delta}$-set.

Next, assume that $Y$ is a remainder of $\mathbf{Q}$. Let $Z=A \cup Y$, where $Z$ is completely metrizable, $A \simeq \mathbf{Q}, A \cap Y=\varnothing, A$ is dense in $Z$. It is immediate that $Y$ is a Polish space. Suppose $Y$ is locally compact at some point $p \in Y$. Let. $U_{Y}$ be an open set in $Y$ containing $p, J=\mathrm{Cl}_{Y}\left(U_{Y}\right)$ is compact. Then $J$ is closed in $Z$. There is an open set $U$ in $Z$ such that $U \cap Y=U_{Y}$. Then $U \backslash J$ is open in $Z$ and therefore topologically complete. But $U \backslash J$ is a subset of $A$ with no isolated point, so $U \backslash J \simeq \mathbf{Q}$, a contradiction.

For the sake of completeness, we include the following. The proofs are immediate.

Theorem 4. The topological completions of $\mathbf{Q}$ and those of $\mathbf{P}$ are the Polish spaces with no isolated points.

Since $\mathbf{Q}$ is a completion remainder of $\mathbf{P}$, the irrationals, one might wonder whether every $\sigma$-compact metric space is a completion remainder of $\mathbf{P}$. That this is not the case is shown in

Theorem 5. If the metrizable space $S$ contains a nondegenerate continuum then $\mathbf{Q} \times S$ is not a completion remainder of $\mathbf{P}$.

Proof. We may assume that $S$ is separable. Let $I$ be a nondegenerate continuum in $S$. Suppose $\boldsymbol{\Theta}: \mathbf{Q} \times S \rightarrow Z$ is an embedding, where $Z$ is a Polish space. We will prove the theorem by showing that $Z \backslash \Theta(\mathbf{Q} \times S)$ contains a nondegenerate connected set and hence is not homeomorphic to $\mathbf{P} . Z$ is a 
dense $G_{\delta}$-set in some compact metric space $K$. Let $K \backslash Z=\bigcup_{n<\omega} K_{n}$, where each $K_{n}$ is compact. For each $t \in \mathbf{R}$, let

$$
L_{t}=\bigcap_{n<\omega} \mathrm{Cl}_{K}\left(\boldsymbol{\Theta}\left(\left(t-2^{-n}, t+2^{-n}\right) \cap \mathbf{Q}\right) \times I\right) .
$$

Note that if $t \in \mathbf{Q}$ then $L_{t}=\Theta(\{t\} \times I)$, and if $t \notin \mathbf{Q}$ then $L_{t} \cap \boldsymbol{\Theta}(\mathbf{Q} \times S)=\varnothing$.

Let $W_{n}=\left\{t \in \mathbf{R}: L_{t} \cap K_{n} \neq \varnothing\right\}$. Suppose some $W_{k}$ is dense in some open interval $(a, b)$ in $\mathbf{R}$. Let $q \in(a, b) \cap \mathbf{Q}$, and let $t_{n} \in W_{k}$ with $t_{n} \rightarrow q$. Let $x_{n} \in L_{t_{n}} \cap K_{k}$. There is a limit point $x$ of $\left\{x_{n}: n \in \omega\right\}$ in $K_{k}$. Clearly $x \in L_{q}=\boldsymbol{\Theta}(\{q\} \times I) \subset Z$, a contradiction. Thus each $W_{k}$ is nowhere dense.

Now pick distance points $s$ and $p$ of $I$, pick $q \in \mathbf{Q}$, and let

$$
\delta=\rho_{K}(\boldsymbol{\Theta}(q, p), \boldsymbol{\Theta}(q, s)),
$$

where $\rho_{K}$ is a metric on $K$. Note that $M=\left\{r \in \mathbf{Q}: \rho_{K}(\Theta(r, p), \Theta(r, s))>\right.$ $\delta / 2\}$ is a nonempty open subset of $\mathbf{Q}$. Let $t \in \bar{M} \backslash\left(\mathbf{Q} \cup \bigcup_{n<\omega} W_{n}\right)$. Then $L_{t} \cap$ $K_{n}=\varnothing$ for all $n<\omega$, i.e., $L_{t} \subset Z$. Since $t \notin \mathbf{Q}, L_{t} \subset Z \backslash \Theta(\mathbf{Q} \times S)$. Choose $q_{n} \in M, q_{n} \rightarrow t$. By passing to a subsequence if necessary, we may assume that $\left\{\boldsymbol{\Theta}\left(\left\{q_{n}\right\} \times I\right): n \in \omega\right\}$ converges in the Vietoris topology on $2^{K}$ to some set $J$, which is nondegenerate, connected, and contained in $L_{t}$. Thus $Z \backslash \Theta(\mathbf{Q} \times S)$ contains a nondegenerate connected set and so is not homeomorphic to $\mathbf{P}$.

Corollary 4. $\mathbf{Q} \times \mathbf{R}$ is not a completion remainder of $\mathbf{P}$.

Remark. The following generalization has essentially the same proof as that of Theorem 5.

Theorem 6. If $X$ contains a closed subset $Y$ such that there exists an open and closed mapping $f: Y \rightarrow \mathbf{Q}$ such that each $f^{-1}(q)$ is a nondegenerate continuum, then $X$ is not a completion remainder of $\mathbf{P}$.

Theorem 7. Every $\sigma$-compact, 0 -dimensional metrizable space is a completion remainder of $\mathbf{P}$.

Proof. Suppose $Y$ is as in the hypothesis. There is an embedding $\phi: Y \rightarrow$ P. $\mathbf{P} \backslash \phi(Y)$ is a $G_{\delta}$-set in $\mathbf{P}$; it is separable, 0-dimensional, metrizable, and nowhere locally compact, so it is homeomorphic to $\mathbf{P}$.

\section{Completion Remainders of $\mathbf{Q}(\kappa)$ AND $\mathbf{P}(\kappa)$}

Throughout this section $\kappa$ denotes an infinite cardinal. Let $\mathbf{Q}(\kappa)$ denote a $\sigma$-discrete metric space in which every open set has cardinality $\kappa$. Medvedev [2] has shown that all such spaces are homeomorphic. Let $\mathbf{P}(\kappa)$ denote a complete metric space with covering dimension 0 that has density $\kappa$ and local density $\kappa$ at each point and that is nowhere locally $\kappa$-compact. A straightforward argument shows that all such spaces are homeomorphic; in particular, $\mathbf{P}(\kappa)$ is homeomorphic to the Baire space $B(\kappa)$, the countable Cartesian product of discrete spaces of cardinality $\kappa$. It follows that $\mathbf{P}(\kappa)$ is a completion remainder of $\mathbf{Q}(\kappa)$. The 0-dimensionality, however, is not necessary. We have

Theorem 8. The completion remainders of $\mathbf{Q}(\kappa)$ are the completely metrizable spaces that have density $\kappa$ and local density $\kappa$ at every point but that are nowhere locally $\kappa$-compact. 
Before proving Theorem 8 we present a lemma that extends the old result of Niemytzki and Tchyonoff [4] that a metrizable space is compact if and only if every compatible metric on the space is complete.

Lemma 2. Let $X$ be a metrizable space. Then the following are equivalent.

(A) $X$ is nowhere locally compact.

(B) $X$ can be embedded in a metrizable space $Z$ in such a way that both $X$ and $Z \backslash X$ are dense in $Z$.

(C) $X$ admits a compatible metric that is nowhere locally complete.

Proof. (B) $\Rightarrow(\mathrm{C})$ Let $X$ and $Z$ be as in (B); $Z$ can be densely embedded in a complete metric space $\langle W, \rho\rangle$. Then $X$ is dense in $W$, and if $\rho_{X}$ denotes the restriction of $\rho$ to $X \times X$ then $X$ is nowhere locally complete according to $\rho_{X}$.

(C) $\Rightarrow(\mathrm{A})$ The proof follows immediately from the Niemytzki-Tychonoff Theorem.

(A) $\Rightarrow$ (B) Suppose $X$ is nowhere locally compact. Let $\rho$ be a metric on $X$. We make repeated use of the following observation.

(*) For every nonempty open set $U$ in $X$, there is a sequence $\left\{U_{n}: n<\omega\right\}$ of nonempty open sets in $X$ such that $\overline{U_{0}} \subset U, \overline{U_{n+1}} \subset U_{n}$ for all $n<\omega$, and $\bigcap_{n<\omega} U_{n}=\varnothing$.

There exists a locally finite open cover $G_{0}$ of $X$ such that if $g \in G_{0}$ then $\rho$-diam $g<2^{-0}$ and $g$ contains a point not in $\bar{h}$ for any $h \in G_{0} \backslash\{g\}$.

For each $g \in G_{0}$ let $U_{g}$ be a nonempty open set such that $\bar{U}_{g} \subset g$ and $\bar{U}_{g} \cap \bar{h}=\varnothing$ for every $h \in G_{0} \backslash\{g\}$. Let $\left\{U_{n}(g): n<\omega\right]$ be a sequence as in (*), with $\overline{U_{0}(g)} \subset U_{g}$.

Take $G_{0}^{\prime}=G_{0} \cup\left\{U_{n}(g): g \in G_{0}, n<\omega\right\}$. If $x \in X$ there is an open set $v_{0}(x)$ containing $x$ that intersects only finitely many elements of $G_{0}^{\prime}$. Let $V_{0}=\left\{v_{0}(x): x \in X\right\}$.

There exists, for each $n, 0<n<\omega$, collections $G_{n}, G_{n}^{\prime}, V_{n},\left\{U_{g}: g \in\right.$ $\left.G_{n}\right\},\left\{U_{m}(g): g \in G, m<\omega\right\}$ such that

(1) $G_{n}$ is a locally finite open cover of $X$ and $\rho$-diam $g<2^{-n}$ for all $g \in G_{n}$.

(2) $G_{n}$ refines both $G_{n-1}^{\prime}$ and $V_{n-1}$.

(3) If $g \in G_{n}$ then $U_{g}$ is a nonempty open set such that

(i) $\bar{U}_{g} \subset g$ and $\bar{U}_{g} \cap \bar{h}=\varnothing$ for every $h \in G_{n} \backslash\{g\}$;

(ii) if $h \in G_{0} \cup \cdots \cup G_{n-1}$ and $U_{g} \cap U_{m}(h) \neq 0, m<\omega$, then $\bar{U}_{g} \subset U_{m}(h)$; and

(iii) if $h \in G_{0} \cup \cdots \cup G_{n-1}$ then there is an $m<\omega$ such that $\bar{U}_{g} \cap$ $\overline{U_{m}(h)}=\varnothing$.

(4) If $g \in G_{n}$ then $\left\{U_{m}(g): m<\omega\right\}$ is as in (*) with $\overline{U_{0}(g)} \subset U_{g}$.

(5) $G_{n}^{\prime}=G_{n-1}^{\prime} \cup G_{n} \cup\left\{U_{m}(g): g \in G_{n}, m<\omega\right\}$,

(6) $V_{n}$ is an open cover of $X$ no element of which intersects infinitely many elements of $G_{n}^{\prime}$.

Let $A=\bigcup_{n<\omega} A_{n}$, where $A_{n} \cap A_{m}=\varnothing$ for $n \neq m, A \cap X=\varnothing$, and, for each $n,\left|A_{n}\right|=\left|G_{n}\right|$. Let $\phi_{n}: A_{n} \rightarrow G_{n}$ be a bijection. Let $Z=A \cup X$. For $V$ open in $X$ let $A_{V}=\left\{a \in A\right.$ : for some $m, n<\omega, a \in A_{n}$, and $\left.\mathrm{Cl}_{X}\left(U_{m}\left(\phi_{n}(a)\right)\right) \subset V\right\}$, and let $E(V)=V \cup A_{V}$. We observe that $\{E(V): V$ open in $X\}$ is a cover of $Z$ and that if $V$ and $W$ are open in $X$ then 
$E(V \cap W)=E(V) \cap E(W)$. Therefore, $\{E(V): V$ open in $X\}$ is a basis for a topology $\Omega$ on $Z$. We also observe that $E(V) \subset E(W)$ whenever $V \subset W$ and that $\mathrm{Cl}_{Z}(E(V))=\mathrm{Cl}_{Z}(V)$ for all open $V$ in $X$. It is easily seen that $\Omega$ is a Hausdorff topology on $Z$. We list three more observations that are useful in showing $\Omega$ is regular.

(1) $\mathscr{U}=\left\{U_{m}(a): m<\omega, a \in A\right\}$ is non-Archimedean in the sense that if two members of $\mathscr{U}$ intersect then one is a subset of the other.

(2) If $p \in A_{n}$ and $q \in A \cap \mathrm{Cl}_{Z}\left(U_{m}\left(\phi_{n}(p)\right)\right)$ then $q \in E\left(U_{m}\left(\phi_{n}(p)\right)\right)$.

(3) If $p \in A_{n}$ then $\mathrm{Cl}_{Z}\left(E\left(U_{m+1}\left(\phi_{n}(p)\right)\right)\right) \subset E\left(U_{m}(\phi(p))\right)$.

Next, assume $p \in A$ and $E(U)$ is a basic open set containing $p$. There is an $n$ such that $p \in A_{n}$. There is an $m$ such that $\mathrm{Cl}_{X}\left(U_{m}\left(\phi_{n}(p)\right)\right) \subset U$. Let $V=E\left(U_{m+1}\left(\phi_{n}(p)\right)\right)$. Then by (3) above, $\mathrm{Cl}_{Z}(V) \subset E\left(U_{m}\left(\phi_{n}(p)\right)\right) \subset E(U)$. Therefore, $\Omega$ is regular at points of $A$.

Next, assume $p \in X$ and $E(U)$ is a basic open set containing $p$. There is an $n$ such that if $p \in g \in G_{n}, h \in G_{n}$, and $g \cap h \neq \varnothing$, then $h \subset U$. Choose an element $g$ of $G_{n}$ containing $p$. For each $i \leq n$ there is an $m_{i}<\omega$ such that $p \notin \mathrm{Cl}_{X}\left(U_{m_{i}}\left(\phi_{i}(a)\right)\right)$ for any $a \in A_{i}$. There is an open set $V$ in $X$, with $p \in V \subset g$, and $\mathrm{Cl}_{X}(V) \cap \mathrm{Cl}_{X}\left(U_{m_{i}}\left(\phi_{i}(a)\right)\right)=\varnothing$ for all $a \in A_{0} \cup \cdots \cup A_{n}$. So, if $a \in A_{0} \cup \cdots \cup A_{n}$, then $a \notin \mathrm{Cl}_{Z}(V)$. Suppose $a \in A_{k}, k>n$, and $a \in \mathrm{Cl}_{Z}(E(V))=\mathrm{Cl}_{Z}(V)$. Then $\left.V \cap E\left(U_{0}(a)\right)\right) \neq \varnothing$ and $U_{0}\left(\phi_{k}(a)\right)$ is a subset of some $h$ in $G_{n}$, and $h \cap g \neq \varnothing$; so $h \subset U$, which implies $a \in E(U)$. Therefore, $\Omega$ is regular at points of $X$.

Next, we exhibit a $\sigma$-locally finite basis for $\Omega$. Let $\Sigma_{0}=\left\{E(g): g \in G_{0}\right\}$. Then $\Sigma_{0}$ is locally finite. For $1 \leq n<\omega$ and $k<\omega$, let $\Sigma(n, k)=\{E(g)$ : $g \in G_{n}, U_{k}(h) \cap g=\varnothing$ for all $\left.h \in G_{0} \cup \cdots \cup G_{n-1}\right\}$. Then $\Sigma(n, k)$ is locally finite. Moreover, if $\Sigma_{n}=\left\{E(g): g \in G_{n}\right\}$, then $\Sigma_{n}=\bigcup_{k<\omega} \Sigma(n, k)$.

Similarly, it follows that for all $m, n<\omega, \Delta_{m, n}=\left\{E\left(U_{m}(g)\right): g \in G_{n}\right\}$ is $\sigma$-locally finite.

Then $\left(\bigcup_{n<\omega} \Sigma_{n}\right) \cup\left(\bigcup_{m, n<\omega} \Delta_{m, n}\right)$ is a $\sigma$-locally finite basis for $\Omega$, so that $(Z, \Omega)$ is metrizable by the Nagata-Smirnov theorem. 2.

Clearly, $A$ is dense in $Z$ and so is $X$. This completes the proof of Lemma

We now return to the proof of Theorem 8 . Note that Theorem 3 is Theorem 8 in the special case $\kappa=\omega$. From now on we assume $\kappa>\omega$.

Assume $Y$ is a completely metrizable space with density $\kappa$ and local density $\kappa$ at each point. It follows directly from Lemma 1 that $Y$ is nowhere locally $\kappa$-compact and therefore nowhere locally compact. We apply Lemma 2 to get a metrizable space $Z$ such that both $Y$ and $Z \backslash Y$ are dense in $Z$. We may assume that $Z$ is completely metrizable, since it can be densely embedded in a completely metrizable space $Z^{\prime}$, and that both $Y$ and $Z^{\prime} \backslash Y$ are dense in $Z^{\prime}$.

Let $G=\bigcup_{n<\omega} G_{n}$ be a $\sigma$-discrete basis for $Z$, where $\left|G_{n}\right|=\kappa$ and $G_{n}$ is discrete, $n<\omega$.

Since $Y$ is completely metrizable, it is a $G_{\delta}$-set in $Z$; let $\left\{V_{n}: n<\omega\right\}$ be a sequence of open sets in $Z$, with $V_{n} \supset V_{n+1}, \bigcap_{n<\omega} V_{n}=Y$.

For each $n<\omega$, let $A_{n}$ be an Axiom of Choice set for $\left\{\left(g \cap V_{n}\right) \backslash Y: g \in\right.$ $\left.G_{n}\right\}$. Then $A_{n}$ is closed and discrete and $A=\bigcup_{n<\omega} A_{n}$ is $\sigma$-discrete and has density $\kappa$ and local density $\kappa$ at every point. It follows that $A \simeq \mathbf{Q}(\kappa)$. Moreover, $A$ is dense in $Z$. For $n=0$, let $W_{0}=V_{0}$, and for $n>0$, let 
$W_{n}=A_{0} \cup \cdots \cup A_{n-1} \cup V_{n}$. Since each $A_{i}$ is closed and $V_{n}$ is open, $W_{n}$ is a $G_{\delta}$-set in $Z$, so $A \cup Y=\bigcap_{n<\omega} W_{n}$ is a $G_{\delta}$-set in $Z$ and therefore completely metrizable.

Next assume that $Y$ is a completion remainder of $\mathbf{Q}(\kappa)$. Then there exist $A$ and $Z, A \simeq \mathbf{Q}(\kappa), Z$ completely metrizable, $Z=A \cup Y$, and $A \cap Y=\varnothing$. Since $A$ is an absolute $F_{\sigma}, Y$ is a $G_{\delta}$-set in $Z$ and thus completely metrizable. Since $A$ is dense in $Z$, we have $d(Y) \leq d(Z) \leq d(A)=\kappa$. Since $Y$ is dense in $Z$, we have $\kappa=d(A) \leq d(Z) \leq d(Y)$. Therefore, $d(Y)=\kappa$. Similarly, $l d_{p}(Y)=\kappa$ for each $p \in Y$. It follows from Lemma 1 that $Y$ is nowhere locally $\kappa$-compact.

Theorem 9. If $Y$ is metrizable, $\operatorname{dim} Y=0$, and $Y$ is the union of countably many sets, each the union of a discrete collection of compact sets, then $Y$ is a completion remainder of $\mathbf{P}(\kappa)$.

Proof. The proof is very similar to that of Theorem 6. Firstly, we know that there is an embedding $\Phi: Y \rightarrow \mathbf{P}(\kappa)$. Secondly, $\mathbf{P}(\kappa) \backslash \Phi(Y)$ is a $G_{\delta}$-set in $\mathbf{P}(\kappa)$; it has covering dimension 0 and density $\kappa$ and local density $\kappa$ at every point and is nowhere locally $\kappa$-compact, so it is homeomorphic to $\mathbf{P}(\kappa)$.

\section{OPEN QUESTIONS}

Question 1. What are the completion remainders of $\mathbf{P}(\kappa)$ ? We do not have a characterization even in case $\kappa=\omega$.

Question 2. In the class of Moore spaces, what are the completion remainders of $\mathbf{Q}$ or of $\mathbf{Q}(\kappa)$ ?

\section{ACKNOWLEDGMENTS}

We are indebted to I. Reclaw, S. Baldwin, and the referee for helpful suggestions.

\section{REFERENCES}

1. R. Engelking, General topology, Revised and completed edition, Sigma Ser. Pure Math., vol. 6, Helderman Verlag, Berlin, 1989.

2. S. V. Medvedev, Topological characteristics of spaces $\mathbf{Q}(\kappa)$ and $\mathbf{Q} \times B(\kappa)$, Moscow Univ. Math. Bull. 41 (1986), 42-45.

3. R. L. Moore, A set of axioms for plane analysis situs, Fund. Math. 25 (1935), 13-28.

4. V. Niemytzki and A. Tychonoff, Beweis des Satzes dass ein metrisierbarer Raum dann und nur dann kompact ist, wenn er in jeder Metrik volständig ist, Fund. Math. 12 (1928), 118120.

5. A. Wilanski, Advanced problem 5602, Amer. Math. Monthly 75 (1968), 685 [Solution by M. D. Mavinkurve 76 (1969), 569].

(B. Fitzpatrick, Jr., and G. F. Gruenhage) Division of Mathematics, FAT, Auburn UniverSity, Auburn, Alabama 36849-5310

E-mail address: GGRUEN@DUCVAX.auburn.edu

(J. W. Ott) Triangle Systems, Inc., Chapel Hill, North Carolina 27709 